\title{
PRINCIPIOS, CRITERIOS E INDICADORES DE SUSTENTABILIDAD PARA PLANTACIONES FORESTALES COMERCIALES DE RÁPIDO CRECIMIENTO
}

\author{
PRINCIPLES, CRITERIA AND INDICATORS FOR SUSTAINABILITY OF COMMERCIAL FOREST \\ PLANTATIONS WITH FAST GROWING SPECIES
}

\author{
Claudia A. Rivera Ríos ${ }^{1}$, Aurelio M. Fierros González ${ }^{*}$, Verónica Vázquez García ${ }^{2}$, Armando Gómez \\ Guerrero $^{1}$ y Alejandro Velázquez Martínez ${ }^{1}$
} ${ }^{1}$ Programa Forestal y ${ }^{2}$ Programa de Desarrollo Rural, Colegio de Postgraduados-Campus Montecillo. Km. 36.5 Carr. México-Texcoco. Montecillo, Texco-
co, Estado de México. Tel. 01 (55) 5804-5900 ext. 1477.

*Autor para correspondencia (amfierros@colpos.mx)

\section{RESUMEN}

Para hacer operativo el concepto de desarrollo sustentable es necesario utilizar principios, criterios e indicadores (PCI) que abarquen los aspectos sociales, ambientales y económicos de la gestión de los recursos forestales. Las plantaciones forestales comerciales son ecosistemas particulares diferentes de los bosques naturales, que requieren de PCI's particulares, que en México no han sido desarrollados. Además, estos PCI's servirán para generar conjuntos específicos para este tipo proyectos. Para obtener un conjunto genérico de PCI's aplicables a programas de plantaciones forestales comerciales, un grupo multidisciplinario de expertos calificó los criterios propuestos por el Centro Internacional de Investigación Forestal en una escala del 1 al 9 ("ranking") y en forma relativa de 0 a 100 \% ("rating"). El análisis se complementó con un taller presencial al que asistieron expertos académicos de diversas áreas, y los resultados se analizaron y discutieron en trabajo de gabinete. Se obtuvo un conjunto compuesto por cinco principios, 25 criterios y 82 indicadores, que integró aspectos ambientales, económicos, sociales, legales, institucionales y de planificación. Este conjunto también puede aplicarse de manera emergente para evaluar directamente el nivel de sustentabilidad de un programa de plantaciones forestales comerciales en particular. lidad.

Palabras clave: Manejo forestal sustentable, principios de sustentabi-

\section{SUMMARY}

An operational concept posed for sustainable development requires the utilization of principles, criteria and indicators (PCI) that should include environmental, social and economical issues for the management of forest resources. The commercial forest plantations are particular ecosystems that clearly differ from natural forests and require of particular PCI's; in México these tools has not been developed yet. These PCI's would be useful for developing specific sets for this particular type of projects. In order to obtain a generic group of PCI's useful for commercial forest plantations, a multidisciplinary group of experts analyzed the criteria proposed by the Center for International Forestry Research, in order to rank them from 1 to 9, and also for rating them from 0 to $100 \%$. This analysis was supplemented by one workshop performed with persons of different academic disci- plines. The information was analyzed and the result was a set of five principles, 25 criteria and 82 indicators which included environmental, economic, social, legal, institutional and planning issues. The resulting set might also be applied, in an emergent way, for a direct evaluation of sustainability in a particular commercial forestry plantation.

Index words: Sustainable forest management, principles of sustainability.

\section{INTRODUCCIÓN}

Con las reformas al Artículo 27 constitucional en 1992, fue posible crear empresas mercantiles con aportaciones de terrenos privados, ejidales y comunales. Estas empresas podrían conjuntar hasta 20000 ha, superficie adecuada para establecer plantaciones forestales comerciales (PFC) (Musálem, 2006), que se caracterizan por el manejo de superficies relativamente grandes. Estas reformas constituyen un mayor estímulo a las plantaciones forestales comerciales, además de que la Comisión Nacional Forestal (CONAFOR), primero a través del Programa de Plantaciones Forestales Comerciales (PRODEPLAN) y luego del Programa ProÁrbol, otorga incentivos para el desarrollo forestal sustentable a través de subsidios directos e de incentivos fiscales. Algunas empresas están realizando proyectos en extensiones relativamente pequeñas mientras que otras manejan extensiones mayores. Diversas empresas están plantando especies de rápido crecimiento, como los eucaliptos (Eucalyptus spp).

Científicos como Guerrero et al. (1999) y Barkin (1998), indican que las plantaciones comerciales de especies de rápido crecimiento significan monocultivos que irán reemplazando la biodiversidad y generarán impactos negativos, sobre todo en lo social y ambiental. En contraste, otras opiniones indican que las plantaciones pueden ser 
benéficas para mejorar el ambiente, aliviar la presión sobre los bosques naturales, aprovechar tierras degradadas y generar empleos, entre otros beneficios (Fierros, 1998). En cualquier caso se desconoce si el manejo de plantaciones atiende correctamente los temas ambientales y socioeconómicos, que son importantes para lograr la sustentabilidad del recurso a largo plazo (Muhtaman et al., 2000).

Según Álvarez y Viveros (1996), el desarrollo de las PFC en México, requiere transitar al desarrollo sustentable. Desafortunadamente, el definir el concepto de desarrollo sustentable puede ser una tarea complicada y subjetiva, e incluso caer en la retórica (Alemán, 2005). En la actualidad, el desarrollo sustentable aparece en el discurso político internacional como una forma de conciliar el crecimiento económico expresado en términos de desarrollo $v s$. el equilibrio del ecosistema, lo que implica mantener una capacidad productiva elevada y a la vez proteger los recursos naturales.

Desde 1992 algunas iniciativas internacionales han buscado métodos que permitan hacer operativo el concepto de sustentabilidad. Entre estos métodos están los principios, criterios e indicadores (PCI), la evaluación de ciclos de vida, los análisis costo-beneficio, el conocimiento basado en el sistema y las evaluaciones de impacto ambiental (Holvoet y Muys, 2004).

Un principio es una verdad o ley fundamental que provee el marco primario necesario para manejar las PFC de manera sustentable. Como criterio se considera a un principio de segundo orden que agrega significado y operacionalidad a un principio, sin que por sí solo sea una medición directa del comportamiento. Un indicador es cualquier variable o componente del ecosistema forestal o sistema de manejo, que se utiliza para inferir el estatus de un criterio en particular (Lammerts y Blom, 1996).

En Asia hay experiencias en el desarrollo de conjuntos de PCI's aplicables a PFC (Muhtaman et al., 2000; Sankar et al., 2000). Sin embargo, en México los esfuerzos para evaluar en este sentido a las PFC han sido prácticamente nulos, lo que plantea la necesidad de contar con un conjunto genérico de PCI's diseñado ex profeso para las PFC desarrolladas en el país, que además sirva como base para generar conjuntos específicos para este tipo proyectos.

En este trabajo se generó un conjunto genérico de PCI's que integra aspectos ambientales, económicos, sociales, legales, institucionales y de planificación, y que sirve para evaluar el grado de sustentabilidad de proyectos de PFC de rápido crecimiento en general, y para desarrollar esta misma herramienta en proyectos específicos.

\section{MATERIALES Y MÉTODOS}

Se seleccionó un conjunto preliminar de PCI's a través del análisis de conjuntos existentes que se han utilizado en la evaluación de la sustentabilidad de bosques naturales, plantaciones forestales y esquemas de certificación. Se consideraron nueve conjuntos de PCI's: CIFOR (Mendoza et $a l .$, 1999), Prueba Norteamericana del CIFOR (Woodley et al., 2000), Prueba CIFOR para plantaciones en Indonesia (Muhtaman et al., 2000), Proceso de Montreal (Proceso de Montreal, 2007), Organización Internacional de Maderas Tropicales (OIMT, 2005), Organización Internacional del Trabajo (OIT, 1997), Proceso de Tarapoto (Tarapoto Proposal, 1995), Fundación Vida para el Bosque (VIBO A. C., 2002) e Instituto Nacional de Investigaciones Forestales Agrícolas y Pecuarias (Flores et al., 2003).

Los conjuntos elegidos se agruparon en una matriz, y se eliminaron los PCI's que evidentemente no aplicaban para PFC, así como los que se repetían. También se ajustó la redacción para hacerla más congruente en el contexto de las PFC. El conjunto preliminar resultante se sometió a una evaluación de gabinete por parte de expertos, cuyo número y especialidades se mencionan en resultados y discusión. Se buscó que el grupo de expertos seleccionado tuviera una composición balanceada de acuerdo con las dimensiones ambiental, productiva, social y económica.

En esta evaluación los expertos calificaron el ranking (del 1 al 9) y el rating (0 a $100 \%$ ) de cada principio, criterio e indicador, según la importancia considerada por cada uno de ellos. Se calculó la suma de los votos de los expertos, tanto en ranking como para en rating, para cada elemento de decisión (principios, criterios o indicadores). Esta suma constituyó el peso total, y una vez que se obtuvo el peso total de cada uno de los elementos de decisión se procedió a obtener el peso relativo. Por ejemplo, el criterio 2.1 perteneciente al principio 2 (Cuadro 3), recibió calificaciones de 9, 7 y 9 mediante la técnica de ranking, y de 40,20 y $25 \%$ mediante la técnica de rating. La suma total para tal criterio es de 25 y $85 \%$ para ambas técnicas, respectivamente. Lo mismo se hace con cada criterio del principio 2 .

Posteriormente se calculó el peso relativo para cada técnica (ranking y rating), al dividir su peso total entre la suma de todos los pesos totales y multiplicar el resultado por cien. Para el ejemplo presentado, si se supone que la suma de los pesos totales de todos los criterios del principio 2 fue de 90 y 301 para cada técnica, el peso relativo del criterio 2.1 mediante la técnica de ranking se calcularía al dividir 25/90 y multiplicarlo por cien. En cambio, para la técnica de rating se dividiría 85/301 y también se multiplicaría por cien. El resultado en este ejemplo daría un peso 
relativo de 27.8 mediante la técnica de ranking y de 28.2 mediante la técnica de rating.

Finalmente se obtuvo el peso combinado para cada elemento de decisión, al promediar los valores obtenidos por ambas técnicas. En el ejemplo anterior, el peso combinado sería el resultado de promediar 27.8 con 28.2 , es decir, 28. Mendoza et al. (1999) describieron el detalle de la metodología de ponderación y procesamiento de estos sistemas.

Una vez que se tuvieron los pesos combinados de todos los parámetros se convocó a un taller presencial de dos días que se llevó a cabo en diciembre de 2006. A los asistentes se les dio a conocer el peso combinado que se obtuvo para cada PCI, y se hizo énfasis en los que obtuvieron mayor o menor peso combinado, a fin de establecer puntos de reflexión. Se llevó a cabo una discusión en torno a la pertinencia de dejar o eliminar a los PCI's calificados. La discusión siempre se dirigió a la obtención definitiva de los PCI's genéricos, que pudieran servir posteriormente para generar una lista específica aplicable a alguna PFC en particular.

Este conjunto fue revisado a profundidad por los autores para detectar inconsistencias, y aplicar una redacción correcta, según Lammerts y Blom (1996). Al considerar nuevamente el marco conceptual y enriquecerlo con referencias de experiencias en PFC y revisión de información, se logró definir al menos un verificador recomendado para cada indicador. En esta etapa también se seleccionaron otros verificadores, que constituyen alguna fuente de datos o información que mejora la especificidad o la evaluación de un indicador.

\section{RESULTADOS Y DISCUSIÓN}

Después de que todos los PCI's de los conjuntos considerados se agruparon en una sola matriz, se enlistaron un total de seis principios, 37 criterios y 242 indicadores. Al revisarse y eliminarse los que no aplicaban para PFC y en el contexto de México, se dejó un conjunto constituido por cinco principios, 27 criterios y 94 indicadores. La redacción se adaptó al contexto de las PFC y se eliminaron los indicadores que eran exclusivos para bosques naturales.

Para llevar a cabo la evaluación de gabinete se convocó a 16 expertos locales, de los cuales respondieron ocho. Aún así se logró una composición balanceada, pues hubo dos expertos en áreas sociales, uno en área económica, dos en aspectos ambientales, uno de manejo forestal, un especialista en PFC y un experto en criterios e indicadores. De esta manera se cumplió con recomendaciones de Mendoza et al. (1999), quienes indicaron que la relevancia y solidez del análisis multicriterio (AMC) depende finalmente del conocimiento y experiencia del equipo experto formado.

Hay dos aspectos que aún podrían mejorarse. El primero con relación a la diversidad de género, pues Mendoza et al. (1999) recomiendan asegurarla tanto como sea posible, y en el presente caso sólo hubo una mujer en el equipo experto. Otro aspecto se refiere a la importancia de incluir diferentes perspectivas en el equipo, como académicos, consultores, miembros de organizaciones no gubernamentales y representantes del gobierno; esto último no se logró pues siete de los ocho expertos eran académicos.

Además, Mendoza et al. (1999) propusieron que el equipo formado debe de conocer bien el esquema jerárquico de PCI, y tener un amplio conocimiento sobre la unidad de manejo forestal en cuestión. Para este caso, lo anterior significaría que los ocho expertos comprenden bien el marco teórico y conceptual, y cuentan con conocimientos relevantes sobre PFC. Pero estos puntos no se cumplieron completamente, ya que al recibir los resultados de los expertos, e incluso durante el tiempo de calificación, surgieron dudas sobre el marco conceptual; además, $75 \%$ del equipo no contaba con conocimientos sólidos en relación a las PFC. Estos dos aspectos fueron los que motivaron la decisión de modificar la metodología de Mendoza et al. (1999), pues únicamente se aplicó el análisis multicriterio, pero no la comparación por pares.

En lugar de la comparación por pares se hizo un taller presencial al que asistieron 10 académicos con enfoques y experiencias muy distintos, lo cual favoreció una mejor discusión en el análisis de los indicadores. Aunque la asistencia no fue constante, se consideró una experiencia enriquecedora y los resultados permitieron obtener los productos esperados. Los pesos combinados proporcionaron puntos de reflexión en aquellos principios, criterios e indicadores con mayor o menor valor, y propuestas para ordenarlos jerárquicamente (Cuadro 1).

El resultado final de este taller fue un conjunto de PCI's que se perfeccionó mediante una revisión final. El conjunto final quedó constituido por cinco principios, cuyos criterios e indicadores pueden consultarse en los Cuadros 2 al 6. El primer principio se refiere al mantenimiento de la integridad del ecosistema; el segundo, al marco económico, legal e institucional en el que se desarrolla el manejo de las plantaciones en México; el tercero se refiere al acceso equitativo a los beneficios generados en la plantación; el cuarto está relacionado con un rendimiento estable y calidad de bienes y servicios derivados de la plantación; y el quinto es relativo a la salud de los trabajadores y comunidades aledañas de la plantación. Los cinco principios 
corresponden a aspectos ambientales, sociales, económicos y de manejo.

El análisis del conjunto aquí desarrollado puede hacerse con relación a los trabajos realizados en México, y con los efectuados internacionalmente para ecosistemas de plantaciones forestales comerciales. En el primer caso es importante destacar que los conjuntos diseñados a nivel de país se han enfocado a bosques naturales. Los estudios a nivel de unidad de manejo han sido desarrollados por iniciativas de la Organización Internacional de Maderas Tropicales, el Instituto Nacional de Investigaciones Forestales, Agrícolas y Pecuarias y el Fondo Mundial para la Naturaleza (WWF, por sus siglas en inglés); sin embargo, la mayoría han sido planteados para bosques naturales, tanto templados como tropicales. En consecuencia, se utiliza un alto número de indicadores ecológicos y sociales, $\mathrm{y}$ en menor grado los de manejo.

Cuadro 1. Pesos combinados de los principios obtenidos por análisis multicriterio (\%).

\begin{tabular}{lccc}
\hline Principio & Peso relativo (ranking) & Peso relativo (rating) & Peso combinado \\
\hline P1 (institucional/legal/económico) & 21 & 23 & 22 \\
P2 (integridad del ecosistema) & $22^{\dagger}$ & $23^{\dagger}$ & $23^{\dagger}$ \\
P3 (acceso equitativo a los recursos y beneficios) & 21 & 21 & 21 \\
P4 (salud de los participantes) & $16^{\dagger \dagger}$ & $14^{\dagger \dagger}$ & $15^{\dagger \dagger}$ \\
P5 (rendimiento y calidad de bienes y servicios) & 20 & 19 & 19 \\
\hline
\end{tabular}

${ }^{\dagger}$ Mayor peso $\mathrm{y}^{\dagger \dagger}$ Menor peso.

Cuadro 2. Criterios e indicadores del principio 1.

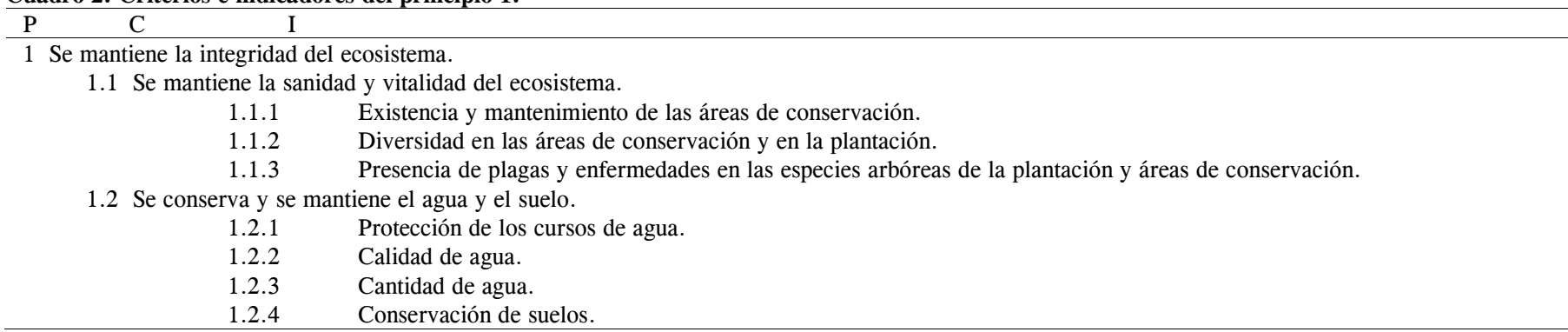

$\mathrm{P}=$ principio; $\mathrm{C}=$ criterio; $\mathrm{I}=$ indicador .

Cuadro 3. Criterios e indicadores del principio 2.

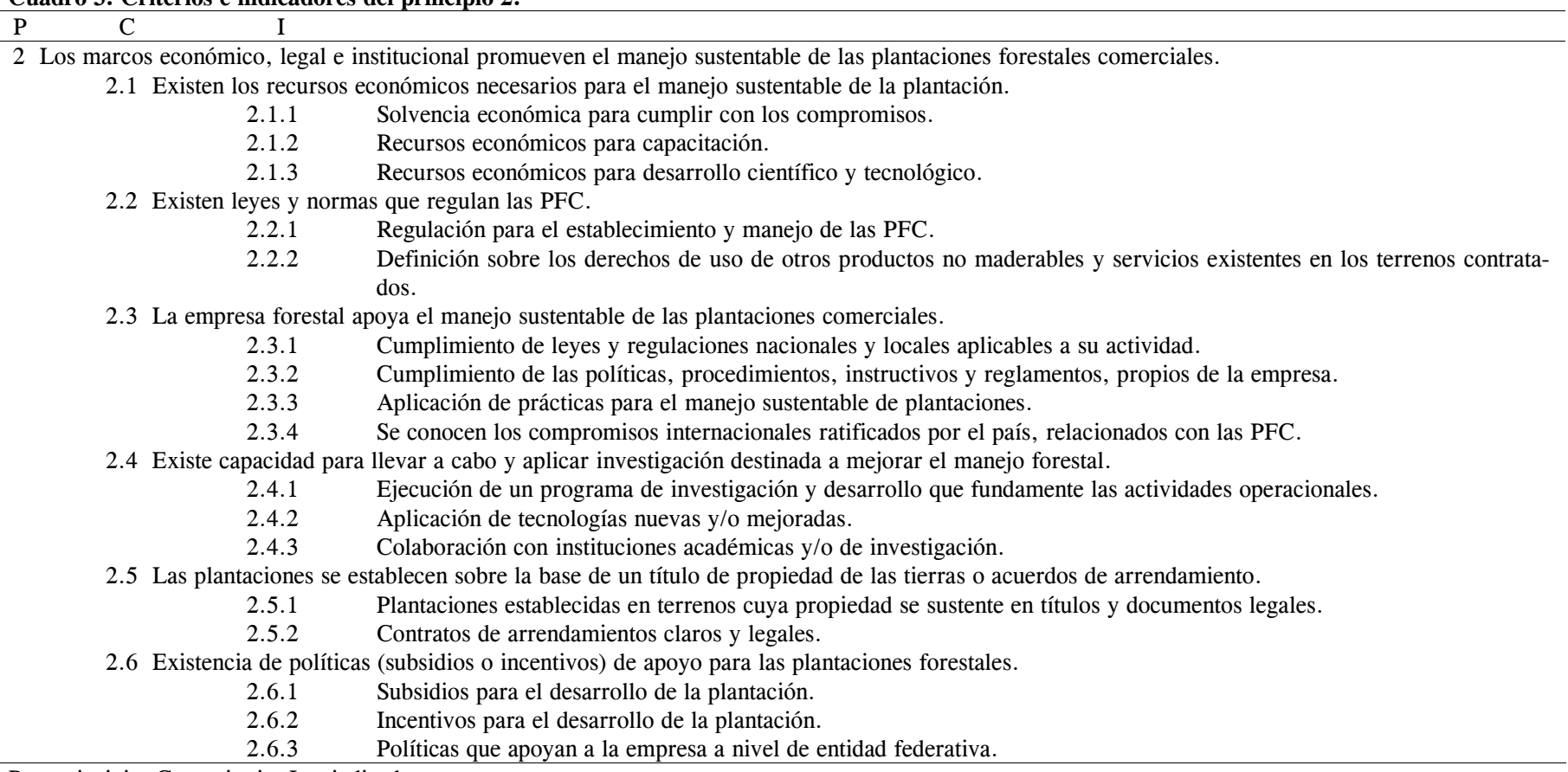

$\mathrm{P}=$ principio; $\mathrm{C}=$ criterio; $\mathrm{I}=$ indicador 
Cuadro 4. Criterios e indicadores del principio 3.

\begin{tabular}{|c|c|c|}
\hline $\mathrm{P}$ & $\mathrm{C}$ & \\
\hline \multirow{2}{*}{\multicolumn{3}{|c|}{$\begin{array}{l}3 \text { La empresa forestal permite un acceso equitativo a los beneficios derivados de la plantación. } \\
\text { 3.1 Existe una distribución razonable de los beneficios económicos que se derivan de la }\end{array}$}} \\
\hline & & \\
\hline & 3.1 .1 & Apego a la normatividad nacional en la asignación de salarios y otras prestaciones. \\
\hline & 3.1 .2 & Competitividad salarial de la empresa. \\
\hline & 3.1 .3 & Política salarial y de prestaciones no discriminante por razones raciales, genéricas, religiosas y políticas. \\
\hline & 3.2 Los habitantes & omunidades cercanas a la plantación tienen oportunidades de empleo y mejoramiento de su calidad de vida. \\
\hline & 3.2 .1 & Los empleados de la plantación pertenecen a las comunidades locales. \\
\hline & 3.2 .2 & La empresa participa en programas de desarrollo de las comunidades cercanas. \\
\hline & 3.2 .3 & Existencia de industria forestal local que utilice materia prima proveniente de la plantación. \\
\hline & 3.2 .4 & Política de contratación no discriminante para los diferentes grupos sociales. \\
\hline & 3.3 Se favorece la & ción de todos los participantes. \\
\hline & 3.3 .1 & Apoyo para la capacitación del personal directivo de la empresa. \\
\hline & 3.3.2 & Apoyo para la capacitación del personal operativo de la empresa. \\
\hline & 3.3 .3 & Reconocimiento de las necesidades de capacitación diferenciadas por grupo social. \\
\hline & 3.4 Los actores soc & cales conocen los usos y beneficios de las plantaciones forestales. \\
\hline & 3.4.1 & Conocimiento del uso y beneficio de las plantaciones por parte de los actores sociales. \\
\hline & 3.4.2 & Existencia de mecanismos para la divulgación de las actividades en las plantaciones entre los grupos interesados. \\
\hline & 3.5 Existen mecani & icaces de comunicación entre los interesados directos en la plantación. \\
\hline & 3.5 .1 & Disponibilidad de la empresa a dialogar con las comunidades. \\
\hline & 3.5 .2 & El personal de la empresa habla una o más de las lenguas locales. \\
\hline
\end{tabular}

$\mathrm{P}=$ principio; $\mathrm{C}=$ criterio; $\mathrm{I}=$ indicador

Los únicos esfuerzos realizados para plantaciones se han efectuado con fines de certificación forestal, como el caso de la Fundación Vida para el Bosque, A. C. (VIBO), la cual tiene un sistema de certificación forestal diseñado para evaluar, auditar y certificar el buen manejo forestal de los bosques, que incluye a plantaciones comerciales, con base en los principios y criterios de manejo forestal establecidos a nivel mundial por el Forest Stewardship Council-Consejo de Manejo Forestal. Los numerosos indicadores de la Fundación VIBO abarcan aspectos legales, ecológicos, sociales y económicos. Desafortunadamente, es un conjunto hecho para certificación, muy amplio y complejo, que si bien contiene principios de importancia en bosques naturales (como los relacionados con la biodiversidad), al aplicarse a plantaciones suelen no reflejar el nivel de sustentabilidad.

A nivel internacional se han desarrollado conjuntos de PCI para certificación, como es el caso de Smartwood (empresa certificadora internacional), y para investigación como los propuestos por el CIFOR. Al comparar el conjunto desarrollado aquí con los trabajos propuestos para sistemas similares, se encuentran similitudes y diferencias. El conjunto de PCI desarrollado para plantaciones industriales en Indonesia (Muhtaman et al., 2000), al igual que el desarrollado aquí, pone menor énfasis en la conservación de la biodiversidad pero presenta un fuerte enfoque en el manejo forestal y en los impactos ambientales. Además, el componente social tiene mayor peso que los componentes ambientales o de manejo, ya que en el conjunto desarrollado aquí hay dos principios relacionados con el componente social (el tercero, referente al acce- so equitativo a los beneficios generados en la plantación; y el quinto, relacionado con la salud de los trabajadores y comunidades aledañas de la plantación).

La diferencia más importante entre el conjunto desarrollado en este trabajo con el propuesto para Indonesia, es que este último presenta criterios e indicadores que acentúan la importancia de los derechos del uso de la tierra. En Indonesia las plantaciones son establecidas en zonas rurales con habitantes indígenas; en México la situación es diferente, pues las plantaciones se establecen preferentemente en terrenos rentados no habitados. Los criterios e indicadores del conjunto aquí propuesto ponen énfasis en el establecimiento de contratos claros y legales, sin considerar los derechos de uso de la tierra por parte de los propietarios, quienes ya se benefician con la renta pagada.

Otro caso de desarrollo de conjuntos para plantaciones comerciales fue realizado en la India por Sankar et al. (2000), en el cual se proponen cuatro principios relacionados a las cuestiones políticas y legales, ecológicas, sociales y de manejo. En la propuesta de la India se da más peso a los principios relacionados con las cuestiones políticas y legales y al aspecto social. Al igual que en el presente trabajo, se menciona la importancia de la equidad de género y el acceso equitativo a los beneficios derivados de la plantación. Pero a diferencia del conjunto de la India, en el que aquí se propone hay menor número de criterios e indicadores relacionados con el manejo de la plantación, lo que pone de manifiesto nuevamente la importancia que dieron los expertos a los indicadores de índole social. 
Cuadro 5. Criterios e indicadores del principio 4.

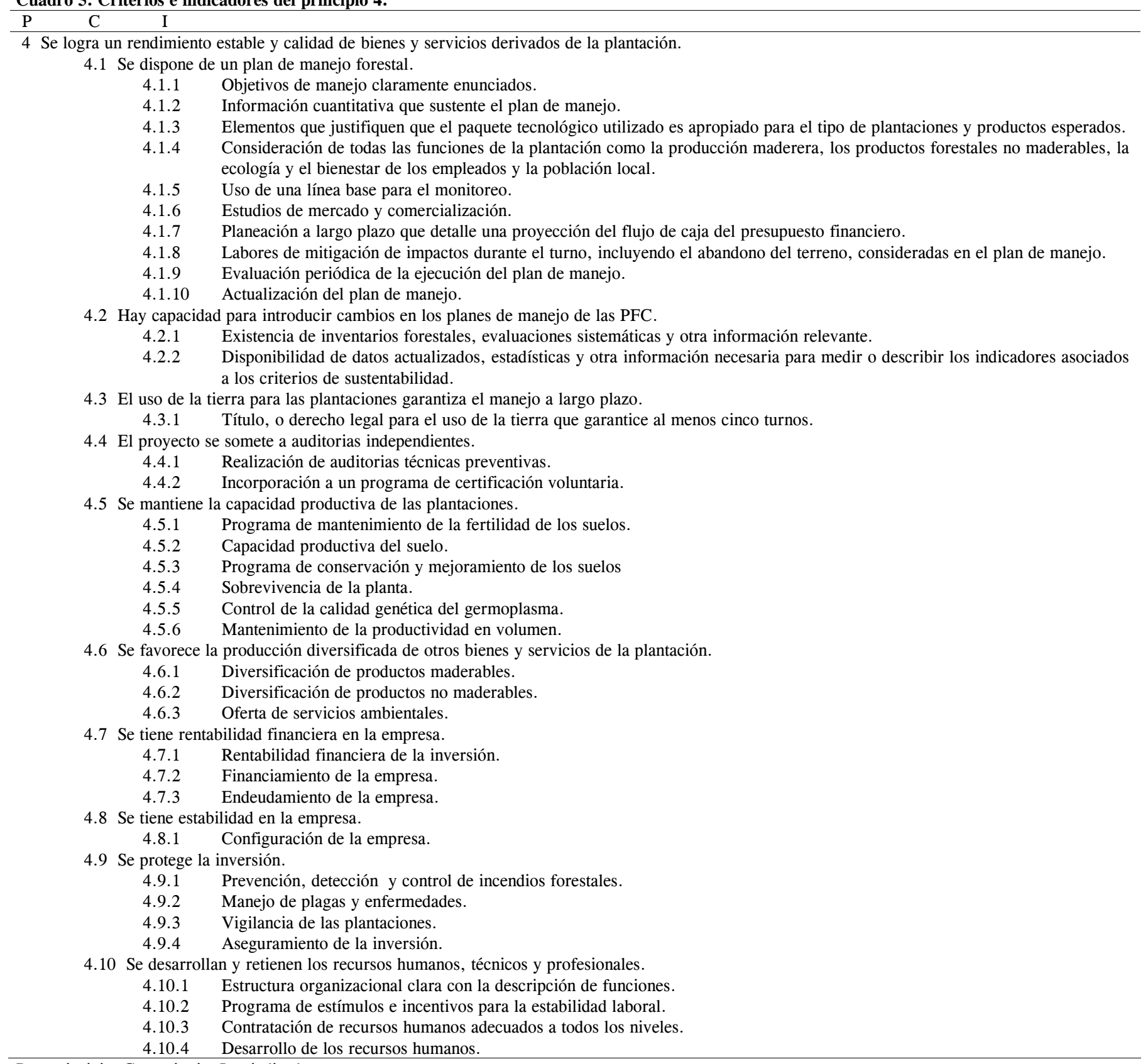

$\mathrm{P}=$ principio; $\mathrm{C}=$ criterio; $\mathrm{I}=$ indicador.

Cuadro 6. Criterios e indicadores del principio 5.

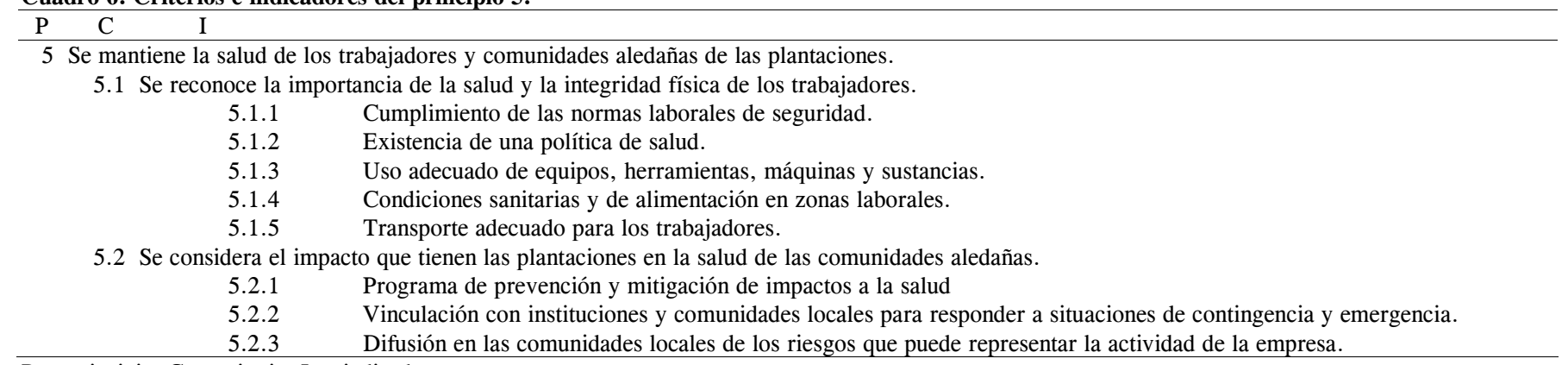

$\mathrm{P}=$ principio; $\mathrm{C}=$ criterio; $\mathrm{I}=$ indicador. 


\section{CONCLUSIONES}

Se desarrolló un conjunto genérico de principios, criterios e indicadores que integra aspectos ambientales, económicos, sociales, legales, institucionales y de planificación. Este conjunto es el primer esfuerzo en México con fines de monitoreo e investigación para plantaciones forestales comerciales, en particular para las de especies de rápido crecimiento. Sirve también para aplicarse de manera emergente en la evaluación del nivel de sustentabilidad con que se manejan proyectos de plantaciones forestales de rápido crecimiento, o bien como base para desarrollar conjuntos para proyectos específicos de plantaciones.

\section{AGRADECIMIENTOS}

Este estudio recibió apoyo financiero del proyecto de investigación del Fondo Sectorial CONAFOR-CONACYT 2005-CO2-14649 "Desarrollo de Criterios e Indicadores para el Manejo Forestal Sustentable en Plantaciones Forestales Tropicales en Oaxaca y Veracruz".

\section{BIBLIOGRAFÍA}

Alemán S (2005) Desarrollo sustentable: Teoría y Práctica. Ecofronteras 24:3-10.

Álvarez-Icaza P, J Viveros (1996) La construcción del modelo mexicano de plantaciones forestales: Un acercamiento ambiental Cuad. Agrarios 14:25-33.

Barkin D (1998) Riqueza, Pobreza y Desarrollo Sustentable. Ed. Jus y Centro de Ecología y Desarrollo. México. ISBN: 9687671041. Disponible en: http://anea.org.mx/publicaciones.htm.

Fierros G A M (1998) Especies exóticas y nativas para el desarrollo de plantaciones forestales comerciales. In: Memoria del ciclo de conferencias "El Sector Forestal de México: Avances y Perspectivas". 29 de Octubre de 1997 al 29 de Abril de 1998. SEMARNAP, México, D. F. pp:45-60.

Flores G, G Moreno, C Quiñones (2003) Selección de criterios e indicadores para evaluar el manejo forestal sustentable. CIR Pacífico Centro, INIFAP. Publicación Esp. No. 2. 72 p.

Guerrero M T, C Reed, B Vegter (1999) La industria forestal y los recursos naturales en la Sierra Madre de Chihuahua: Impactos sociales, económicos y ecológicos. Comisión de Solidaridad y
Defensa de los Derechos Humanos, A. C. y Texas Center for Policy Studies. Chihuahua, México y Texas, U.S.A. 63 p.

Holvoet B, B Muys (2004) Sustainable forest management worldwide: A comparative assessment of standards. Internatl. For. Rev. 6:99-122.

Lammerts V B E, E M Blom (1996) Principios, Criterios e Indicadores. Esquema Jerárquico para la Formulación de Estándares para el Manejo Forestal Sostenible. The Tropenbos Foundation. Holanda. $59 \mathrm{p}$.

Mendoza G A, P Macoun, R Prabhu, D Sukadri, H Purnomo, Hartanto H (1999) Guidelines for Applying Multi-criteria Analysis to the Assessment of Criteria and Indicators. CIFOR, Jakarta, Indonesia. Toolbox Series No 9. $81 \mathrm{p}$.

Muhtaman D, C Siregar, P Hopmans (2000) Criteria and Indicators for Sustainable Plantation Forestry in Indonesia. Center for International Forestry Research. Bogor, Indonesia. $72 \mathrm{p}$.

Musálem M A (2006) Silvicultura de Plantaciones Forestales Comerciales. Universidad Autónoma Chapingo, División de Ciencias Forestales. Departamento de Ecología y Silvicultura. 208 p.

Organización Internacional de Maderas Tropicales, OIMT (2005) Criterios e Indicadores Revisados de la OIMT para la Ordenación Sostenible de los Bosques Tropicales con Inclusión de un Formato de Informes. Serie OIMT de Políticas Forestales No. 15. Yokohama, Japón. 40 p.

Organización Internacional del Trabajo, OIT (1997) Repertorio de Recomendaciones Prácticas sobre Seguridad y Salud en el Trabajo Forestal. Organización Internacional del Trabajo. Ginebra. $85 \mathrm{p}$.

Proceso de Montreal (2007) Criterios e Indicadores para la Conservación y el Manejo Sustentable de los Bosques Templados y Boreales. Disponible en: http://www.rinya.maff.go.jp/mpci/reppub/1995/santiago_s.

Sankar S, P C Anil, M Amruth (2000) Criteria and Indicators for Sustainable Plantation Forestry in India. Center for International Forestry Research. Bogor, Indonesia. 83 p.

Tarapoto Proposal (1995) Proposal of Criteria and Indicators for Sustainability of the Amazon Forests. Results of the Regional Workshop on the Definition of Criteria and Indicators for Sustainability of Amazonian Forests. Tarapoto, Peru, 23-25 Feb. 1995. Pro Tempore Secretariat, Amazon Cooperation Treaty, Lima, Peru. 149 p.

VIBO A C (2002) Estándar Genérico de Manejo Forestal de la Fundación Vibo, A. C. Programa de Certificación Forestal. Durango, México. $21 \mathrm{p}$.

Woodley S G, Alward, G Iglesias, T Hoekstra, B Holt, L Livingston, J Loo, A Skibicki, W Clinton, P Wright (2000) North American Test of Criteria and Indicators of Sustainable Forestry. IMI Report 3, USDA Forest Service, Inventory and Monitoring Institute. Fort Collins, Co, USA. 175 p. 